Original

\title{
Contrast-to-noise ratio difference in small field of view cone beam computed tomography machines
}

\author{
Boulos Bechara1), Clyde A. McMahan²), William S. Moore1), Marcel Noujeim¹), \\ Hassem Geha') and Fabricio B. Teixeira ${ }^{3)}$ \\ 1)Department of Oral and Maxillofacial Radiology, University of Texas Health Science Center \\ at San Antonio, San Antonio, TX, USA \\ 2)Department of Pathology, University of Texas Health Science Center at San Antonio, San Antonio, TX, USA \\ 3)Department of Endodontics, University of Texas Health Science Center \\ at San Antonio, San Antonio, TX, USA
}

(Received 18 April and accepted 4 July 2012)

\begin{abstract}
Each of three cone beam computed tomography (CBCT) machines offer a small field of view (FOV); Promax, Accuitomo 3D, and Kodak 9000. The objectives were to determine, when using a small FOV, the difference in contrast-to-noise ratios (CNR) of images obtained from different machines and different scan settings within each machine. A phantom made of a dry mandible with an epoxy resin based substitute (ERBS) block was used. The ERBS block was fixed to the lingual cortex in the posterior left mandible. Water was used to simulate soft tissue. Twenty scans were acquired. CNR was calculated as the difference in density of the ERBS block and a control area divided by the standard deviation of the difference. The CNR obtained from images acquired with the Promax machine (voxel size of $0.2 \mathrm{~mm}$ ) was higher than the other machines (voxel sizes 0.076 , 0.08 , or $0.125 \mathrm{~mm}$ ) when using a small FOV. The Accuitomo $360^{\circ}$ scan had higher CNR than the Accuitomo $180^{\circ}$ scan due to the doubling of the number of basis images acquired. Smaller voxel size does not necessarily lead to enhanced CNR. Increasing the number of basis images did increase the CNR. ( $J$ Oral Sci 54, 227-232, 2012)
\end{abstract}

Correspondence to Dr. Boulos Bechara, Department of Oral and Maxillofacial Radiology, University of Texas Health Science Center at San Antonio, 7703 Floyd Curl Drive, San Antonio, TX 78229-3900, USA

Tel: +1-210-218-9683

E-mail: boulosbchara@hotmail.com
Keywords: cone beam computed tomography; noise; contrast.

\section{Introduction}

Oral and maxillofacial radiology has developed considerably in the last decade. cone beam computed tomography (CBCT) was introduced as an alternative to computerized tomography $(\mathrm{CT})$ in diagnosing bone pathologies or dysfunctions in the maxillofacial complex $(1,2)$. CBCT is considered to be an accurate imaging modality for dental diagnosis and treatment plan purposes $(3,4)$. Patient's age, gender, medical and dental history, and social situation are always considered when building a treatment plan. Radiological services offered are related to almost all of the dental fields including oral surgery (5), oral medicine $(6,7)$, endodontics (8), periodontology (9), orthodontics (10), and implantology (11).

In the current oral and maxillofacial radiology clinical practice, CBCT machines play a crucial role. Patients currently are having a scan for reasons such as depiction of pathology (12), bone quantity and quality for implant placement (13), follow-up for previously accomplished procedure (14), or orthognatic evaluation (15). The degree to which the image quality differs between the available settings on the CBCT machine is not known. It is the radiologist's duty to decide which setting will be used and that decision is related to many factors and affects directly the image quality.

Volume reconstructions in $\mathrm{CBCT}$ are based on back- 


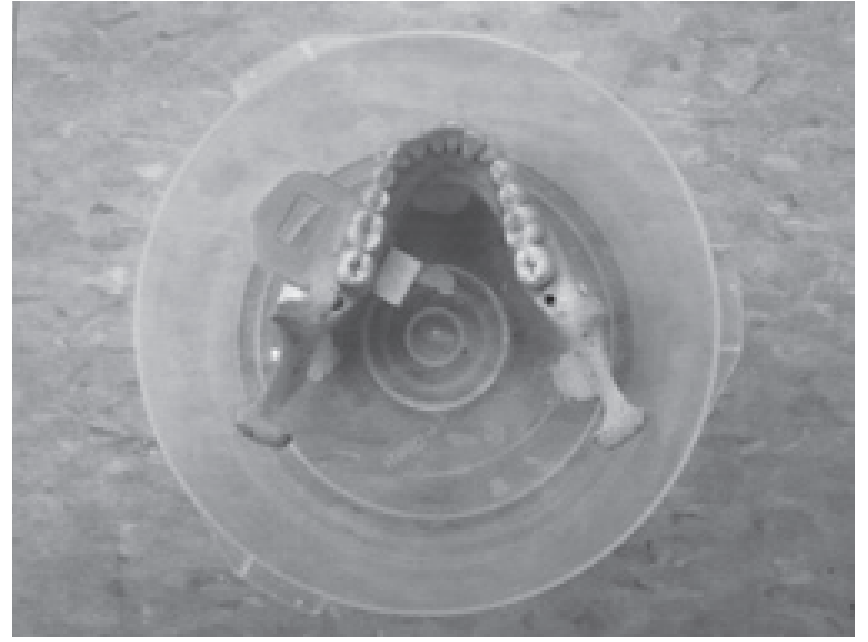

Fig. 1 Phantom used.

projected intensities, obtained from projections located in a full circle around the object under investigation, into the discrete volume grid made up of voxels. The aim of the reconstruction process is to obtain a 'realistic estimate' of the internal structure of an object (16). It was found by many authors that reducing the voxel size will increase the spatial resolution $(17,18)$. Many CBCT machines that offer small voxel sizes are being advertised as providing the best image quality for diagnostic purposes based on an assumption that a smaller voxel size will increase the image quality. An important aspect of image quality in CBCT other than spatial resolution is contrast resolution. Contrast resolution is referred to as the ability of an imaging modality to distinguish between various contrast levels in an acquired image (19). In general, a lesion-tobackground contrast is related to the contrast-to-noise ratio (CNR), and CNR has been considered to be more closely related to image quality than image noise (20). The objective of this study was to determine the degree to which the CNR obtained from images captured using three CBCT machines differs.

\section{Materials and Methods}

The phantom was used in this study (Fig. 1) consisted of a dentate dry mandible with an epoxy resin bone tissue substitute (ERBS) block (21). The mandible was anonymous and taken from the forensics lab of the University of Texas Health Science Center at San Antonio. The block was fixed to the lingual cortex medial to the apical region of the left posterior teeth. Wax was used to standardize and delimit the control area. All structures were surrounded with water to simulate soft tissues. Three machines were used for this study: Promax (Planmeca, Helsinki, Finland), Accuitomo 3D (Morita, Kyoto,
Japan), and Kodak 9000 (Carestream, Rochester, NY, USA). In each machine, two pairs of Kilovolt peak $(\mathrm{kVp})$ and milliampers (mA) settings preset by the manufacturers were chosen. For the Accuitomo, the two pairs of settings were used with both the $180^{\circ}$ scan and the $360^{\circ}$ scan. With the $360^{\circ}$ scan, the number of acquired basis images and patient's radiation exposure are doubled. Two scans for each KVP and mA setting were acquired with a 1 week difference. A total of 16 scans were acquired (Fig. 2). The digital imaging and communications in medicine (DICOM) files were exported and 3D on demand viewer was used to view the acquired volumes. On axial views, a $2 \mathrm{D}$ region of interest (ROI) covering the surface of the ERBS block was chosen and densities along with standard deviations (SD) were computed. On the same view the control area was chosen and the same variables were computed. The settings of the Promax were $84 \mathrm{kVp}$ / $14 \mathrm{~mA}$ and $84 \mathrm{kVp} / 16 \mathrm{~mA}$; the Accuitomo $90 \mathrm{kVp} / 5$ $\mathrm{mA}$ and $90 \mathrm{kVp} / 7 \mathrm{~mA}$; and the Kodak $900070 \mathrm{kVp} / 10$ $\mathrm{mA}$ and $74 \mathrm{kVp} / 10 \mathrm{~mA}$. The exposure times for the $180^{\circ}$ scan and the $360^{\circ}$ scan were $8.5 \mathrm{~s}$ and $17 \mathrm{~s}$ respectively. For the promax scan the exposure time was $18 \mathrm{~s}$ and 14 $\mathrm{s}$ for the Kodak. The volume size used for the promax scans was $4 \times 5 \mathrm{~cm}$, for the Morita $4 \times 4 \mathrm{~cm}$, and for the Kodak $4 \times 5 \mathrm{~cm}$.

\section{Statistical methods}

The CNR was calculated as the difference between the mean ERBS density and the mean control (water) density divided by the standard deviation of the difference. That is,

$$
\mathrm{CNR}=\frac{\text { Mean }_{\text {ERBS }}-\text { Mean }_{\text {Control }}}{\sqrt{S D_{E R B S}^{2}+S D_{\text {Control }}^{2}}}(22,23) \text {. }
$$

Variables were analyzed using analysis of variance (24). The linear model included the effects of machine and settings within machine; within the Accuitomo we also analyzed the effects of scan $\left(180^{\circ}\right.$ and $\left.360^{\circ}\right)$ and voxel size (0.08 and 0.125$)$. Comparisons of means following the analysis of variance were Bonferroni adjusted. Residual analyses indicated that the data were in reasonable conformity with the underlying assumptions of a normal distribution and constant variance. Results are presented as mean \pm standard error (SE).

\section{CNR}

\section{Results}

The CNR for the different machines and settings are shown in Fig. 3 (Panel A). The CNR was significantly $(P \leq 0.0400)$ different between the two settings for the Accuitomo $180^{\circ}$ scan voxel size 0.08 , Accuitomo $180^{\circ}$ scan voxel size 0.125 , and Accuitomo $360^{\circ}$ scan voxel 


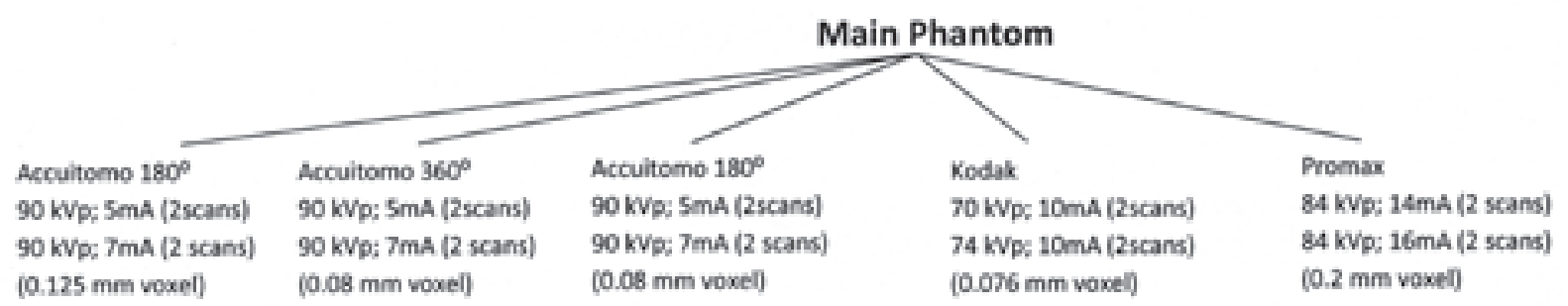

Fig. 2 Study design.
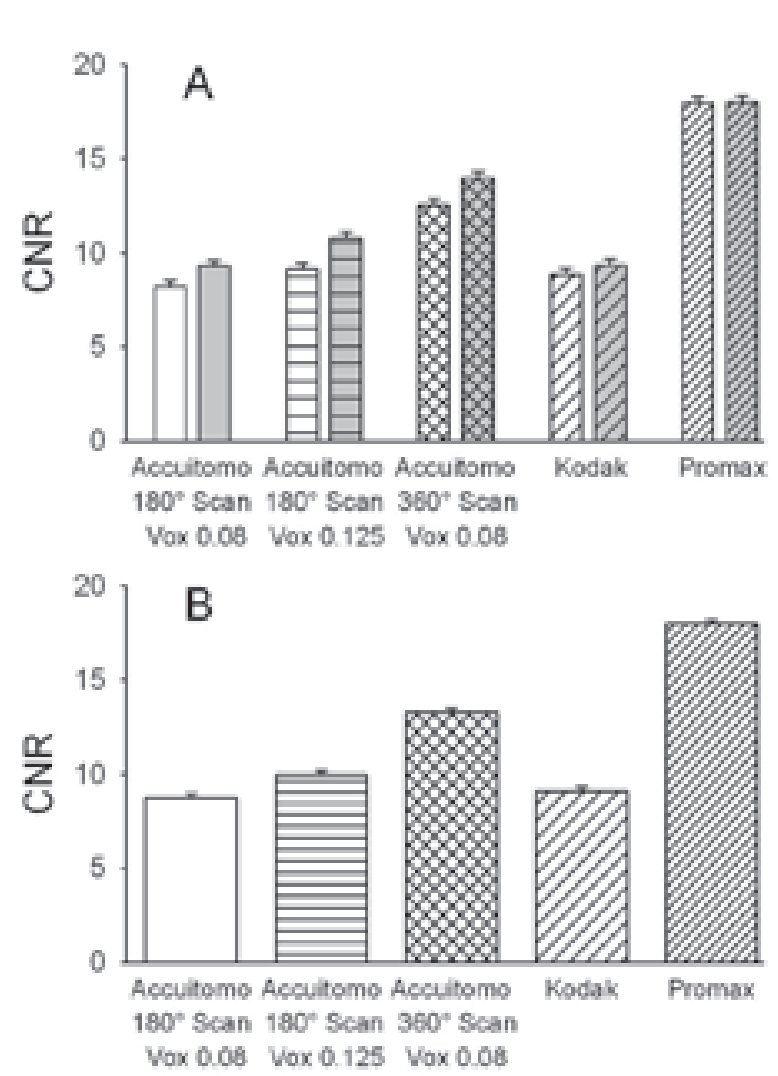

Fig. 3 Panel A. Mean Contrast-to-noise ratio by machine and setting within machine. Error bars represent standard errors. $\square$ Accuitomo $180^{\circ}$ scan Voxel size 0.08 (kVp 90, mA 5), $\square$ Accuitomo $180^{\circ}$ scan Voxel size 0.08 (kVp 90, mA 7), 四 Accuitomo $180^{\circ}$ scan Voxel size 0.125 (kVp 90, mA 5), 巨Accuitomo $180^{\circ}$ scan Voxel size 0.125 (kVp 90, mA 7), Accuitomo $360^{\circ}$ scan Voxel size 0.08 (kVp 90, mA 5), Accuitomo $360^{\circ}$ scan Voxel size 0.08 (kVp 90, mA 7), $\mathbb{Z} / \mathrm{Kodak}(\mathrm{kVp}$ 70, mA 10), $\mathbb{Z}$ Kodak (kVp 74, mA 10), $\mathbb{Z}$ Promax (kVp 84, mA 14), שA Promax (kVp 84, mA 16).

Panel B. Mean Contrast-to-noise ratio by machine, averaged over setting within machine. Error bars represent standard errors. $\square$ Accuitomo $180^{\circ}$ scan Voxel size 0.08 , 国 Accuitomo $180^{\circ}$ scan Voxel size 0.125, Accuitomo $360^{\circ}$ scan Voxel size 0.08 , ZI/ Kodak, $\mathbb{Z}$ Promax.

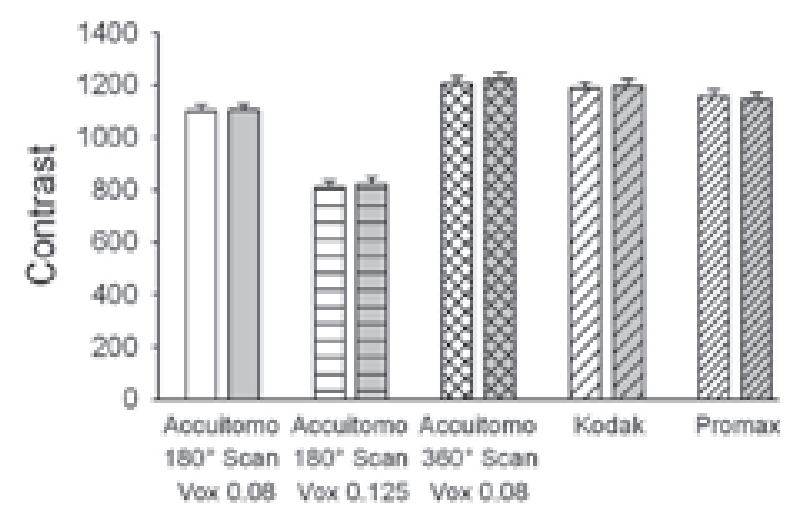

Fig. 4 Mean Contrast by machine and setting within machine. Error bars represent standard errors.

$\square$ Accuitomo $180^{\circ}$ scan Voxel size $0.08(\mathrm{kVp} 90$, $\mathrm{mA}$ 5), $\square$ Accuitomo $180^{\circ}$ scan Voxel size 0.08 (kVp 90, mA 7), 目 Accuitomo $180^{\circ}$ scan Voxel size 0.125 (kVp 90, mA 5), 目Accuitomo $180^{\circ}$ scan Voxel size 0.125 (kVp 90, mA 7), Accuitomo $360^{\circ}$ scan Voxel size 0.08 (kVp 90, mA 5), Accuitomo $360^{\circ}$ scan Voxel size 0.08 (kVp 90, mA 7), Z/ Kodak (kVp 70, mA 10), $\mathbb{Z / \lambda}$ Kodak (kVp 74, mA 10), $\mathbb{Z}$ Promax (kVp 84, mA 14), $\mathbb{Z}$ Promax (kVp 84, mA 16)

size 0.08; the CNR did not differ $(P>0.2942)$ between settings for the Kodak and Promax machines.

The CNR of the machines, averaged over the settings within a machine, are shown in Fig. 3 (Panel B). The CNR for the Accuitomo $180^{\circ}$ scan voxel size 0.08 , Accuitomo $180^{\circ}$ scan voxel size 0.125 , and the Kodak were not significantly different. The Accuitomo $360^{\circ}$ scan voxel size 0.08 had significantly higher CNR than the Accuitomo $180^{\circ}$ scan voxel size 0.08 , Accuitomo $180^{\circ}$ scan voxel size 0.125 , and the Kodak. The CNR for the Promax was significantly higher than the other machines.

\section{Contrast}

The average contrasts are shown in Fig. 4. There were no significant differences between settings within any machine. Averaged over setting, the Accuitomo $180^{\circ}$ scan voxel size 0.125 had significantly lower contrast than the 

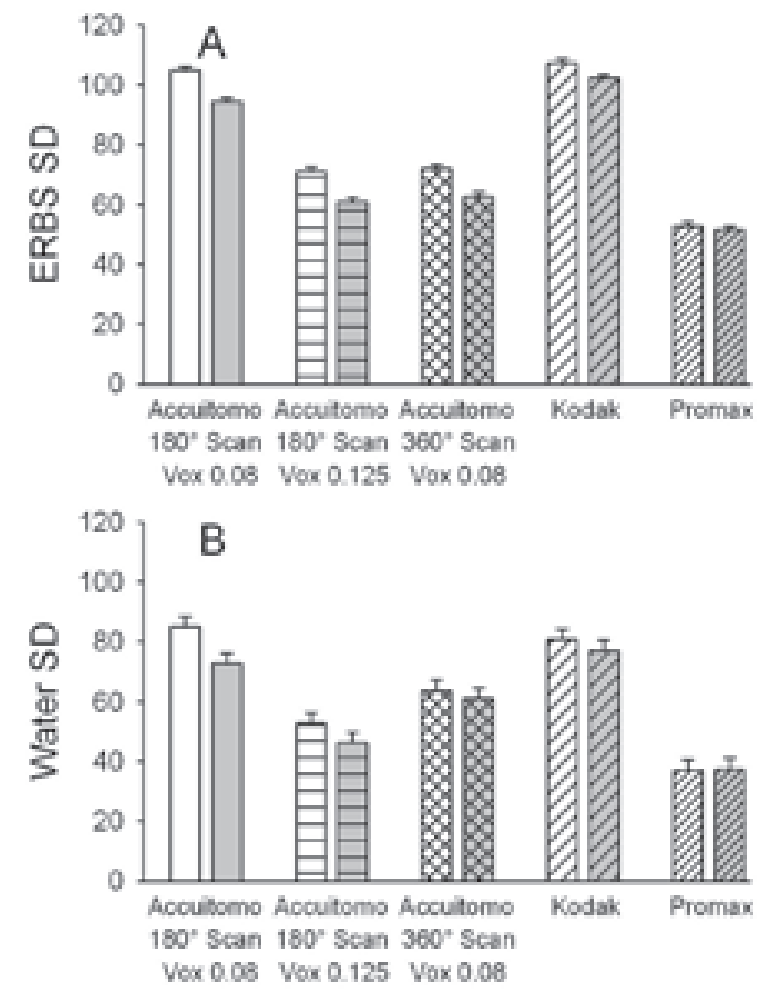

Fig. 5 Panel A. Mean Standard Deviation in ERBS by machine and setting within machine. Error bars represent standard errors.

Panel B. Mean Standard Deviation in water by machine and setting within machine. Error bars represent standard errors.

Accuitomo $180^{\circ}$ scan Voxel size $0.08(\mathrm{kVp} 90$, $\mathrm{mA} 5), \square$ Accuitomo $180^{\circ}$ scan Voxel size $0.08(\mathrm{kVp}$ 90, mA 7), 归 Accuitomo $180^{\circ}$ scan Voxel size 0.125 (kVp 90, mA 5), 国Accuitomo $180^{\circ}$ scan Voxel size 0.125 (kVp 90, mA 7), Accuitomo $360^{\circ}$ scan Voxel size 0.08 (kVp 90, mA 5), Accuitomo $360^{\circ}$ scan Voxel size 0.08 (kVp 90, mA 7), $\ / \triangle$ Kodak (kVp 70, mA 10), $\mathbb{Z}$ Kodak (kVp 74, mA 10), $\mathbb{Z} A$ Promax (kVp 84, mA 14), VA Promax (kVp 84, mA 16).

other machines. The Accuitomo $360^{\circ}$ scan (1214 \pm 18$)$ had a significantly higher contrast than the Accuitomo $180^{\circ} \operatorname{scan}(1101 \pm 18)$.

\section{ERBS SD}

The average ERBS SDs which represents a measure of the noise are shown in Fig. 5 (Panel A). The higher setting had a significantly $(P<0.0286)$ lower SD for all Accuitomo scans and voxel sizes and for the Kodak; there was no difference between the settings for the Promax. Averaged over the settings within a machine, the SD for the Promax $(52.1 \pm 1.0)$ was significantly lower than the other machines; the Accuitomo $180^{\circ}$ scan voxel size $0.125(66.0 \pm 1.0)$ had the next lowest $\mathrm{SD}$ which was significantly lower than the SD for the Accuitomo $180^{\circ}$ scan voxel size 0.08 and the Kodak. The SD for the Accuitomo $360^{\circ}$ scan voxel size $0.08(67.4 \pm 1.0)$ also was significantly lower than the Accuitomo $180^{\circ}$ scan voxel size 0.08 and the Kodak.

\section{Water SD}

The average Water SDs are shown in Fig. 5 (Panel B). For the Accuitomo $180^{\circ}$ scan, the higher setting had a slightly lower $(P=0.0328) \mathrm{SD}$; there was no difference between the settings for the other machines. Averaged over the settings within a machine, the SD for the Promax $(37.2 \pm 2.5)$ was significantly lower than the other machines; the Accuitomo $180^{\circ}$ scan voxel size 0.125 $(49.5 \pm 2.5)$ was significantly lower than the Accuitomo $180^{\circ}$ scan voxel size $0.08(78.8 \pm 2.5)$, the Accuitomo $360^{\circ}$ scan $(62.4 \pm 2.5)$, and the Kodak $(78.9 \pm 2.5)$. The Accuitomo $360^{\circ}$ scan $(62.4 \pm 2.5)$ was significantly lower than the Accuitomo $180^{\circ}$ scan and the Kodak. The Accuitomo $180^{\circ}$ scan voxel size 0.08 and the Kodak were not significantly different.

\section{Discussion}

This study shows that using a smaller voxel size does not necessarily lead to an increased CNR. The SD obtained when smaller voxel sizes were used were significantly increased compared to a 0.2 and 0.125 voxel size; the increased SD resulted in the decrease in the CNR of images acquired when using a voxel size smaller than $0.2(0.076,0.08$, and $0.125 \mathrm{~mm})$. It is important to stress that only small volumes with almost similar dimensions were used in the study.

However, a small voxel size is needed when a higher spatial resolution will help in depicting fine detail. Maloul et al. found that the voxel size had a direct impact on the image resolution and noise (18). Cortical bone thickness and material properties in five thin bone specimens were measured at voxel sizes ranging from 16.4 to $488 \mu \mathrm{m}$. The measurements derived from large voxel size scans showed large increases in cortical thickness (61.9-252.2\%) and large decreases in scan intensity (12.9-49.5\%). Bauman et al., in evaluation of the presence of a second mesio-buccal (MB) root canal, found that the larger the voxel size, the less resolution that will be obtained (17). Overall, $92 \%$ of the maxillary molars had two MB canals upon analysis of horizontal cross-sections with magnification. The CBCT detection of MB canals increased from $60.1 \%$ at $0.4 \mathrm{~mm}$ voxel size to $93.3 \%$ at $0.125 \mathrm{~mm}$ voxel size.

A smaller voxel will not detect as many $\mathrm{x}$-ray photons 
as would a larger voxel size. A decrease in the number of photons acquired by a voxel would result in a decrease in signal leading to an increase in noise as suggested by the lower SDs found in this study for the larger voxel sizes. However, the larger voxel sizes give less spatial resolution and should not be used if the reason of the scan is the depiction of fine details such as depicting a mesio-buccal root canal in a maxillary molar tooth.

Factors other than voxel size contribute to the resultant CNR. All the volume sizes used in this project were small. Other factors include the detector quality and the reconstruction algorithm of each machine. In the current project, even with increased exposure time when the $360^{\circ}$ scan was used, the CNR was still lower than the images acquired with a $0.2 \mathrm{~mm}$ voxel size.

The Accuitomo $360^{\circ}$ scan gave an increased CNR compared to a $180^{\circ}$ scan. Increasing the number of basis images acquired by the machine turning in a full circle motion instead of half of a circle did lead to a better image quality. When a larger number of basis images are acquired, the exposure time increases. In addition, the amount of data to be stored and processed will increase also, but a higher quality image will be obtained. LofthagHansen et al. have shown that exposure parameters should be adjusted according to diagnostic task (25). The CBCT units used were the 3D Accuitomo (Field of view (FOV) $3 \times 4 \mathrm{~cm}$ ) and 3D Accuitomo FPD (FOVs $4 \times 4$ $\mathrm{cm}$ and $6 \times 6 \mathrm{~cm}$ ) both manufactured by J Morita, Kyoto, Japan; a rotation of $180^{\circ}$ compared to a $360^{\circ}$ which takes twice the time to be acquired, gave good subjective image quality, hence a substantial dose reduction can be achieved without loss of diagnostic information. In addition, Bechara et al. concluded that metal artifacts cause a decrease in CNR $(22,23)$. The increase in CNR found in this study when increasing the basis images in the Accuitomo machine may be used to counteract the effects of metal artifacts.

The difference in CNR was not significant between a $4 \times 4 \mathrm{~cm}$ FOV using a $0.08 \mathrm{~mm}$ voxel (rotation of $180^{\circ}$ ) and a $6 \times 6 \mathrm{~cm}$ FOV using a $0.125 \mathrm{~mm}$ voxel (rotation of $180^{\circ}$ ) which suggests with less reconstruction time almost the same CNR can be obtained. Scout images should always be acquired to make sure that the area of interest will be captured in the scan.

The contrast obtained in the $6 \times 6$ FOV was different than the one obtained with the other machines and with the same machine itself when the $4 \times 4 \mathrm{~cm}$ FOVs were used which implies a definite role of the reconstruction algorithm in computing the densities and contributing to the image quality in general.

Tanimoto et al. evaluated the effects of changing the voxel size on the resolution and noise of CBCT reconstruction images (26). The voxel sizes used for reconstruction were 160,80 , and $40 \mu \mathrm{m}$ using prototype software for the Accuitomo F8 (J. Morita, Kyoto, Japan). The MTF at $21 \mathrm{p} / \mathrm{mm}$ was $0.05,0.12$, and 0.12 for voxel sizes of 160,80 , and $40 \mu \mathrm{m}$, respectively, and the SD of the noise was $10.0,13.8$, and $17.1 \%$ for the same respective voxel sizes. The limit of resolution was determined to be the 80 $\mu \mathrm{m}$ voxel size. When the voxels were smaller, the noise increased. Their findings show that decreasing the voxel size increases the noise which conforms to the results in the current paper. In addition, a voxel size smaller than $0.08 \mathrm{~mm}(0.04 \mathrm{~mm})$ didn't enhance the resolution and resulted in more noise.

This study is an in vitro study and looked only at one aspect of image quality. It evaluated how the CNRs of small FOV differ between CBCT machines using the manufacturers' recommendations for $\mathrm{kVp}$ and $\mathrm{mA}$. It may be interesting to evaluate diagnostic accuracy difference between varying voxel sizes when using small volumes. This is particularly important because smaller voxel sizes are being marketed on the assumption that they will lead to the highest image quality.

In conclusion, for small FOVs acquired in CBCT, a $0.2 \mathrm{~mm}$ voxel size lead to a better contrast resolution compared to voxel sizes of smaller dimensions. Decreasing the voxel size led to an increased noise and decreased CNR because fewer x-ray photons are detected. The contrast and spatial resolutions should be evaluated in parallel when using a smaller voxel sizes in CBCT. Scans acquired with higher basis images yield an enhanced CNR.

\section{References}

1. Arai Y, Tammisalo E, Iwai K, Hashimoto K, Shinoda K (1999) Development of a compact computed tomographic apparatus for dental use. Dentomaxillofac Radiol 28, 245-248.

2. Rigolone M, Pasqualini D, Bianchi L, Berutti E, Bianchi SD (2003) Vestibular surgical access to the palatine root of the superior first molar: "lowdose cone-beam" CT analysis of the pathway and its anatomic variations. J Endod 29, 773-775.

3. Chen Q, Liu DG, Zhang G, Ma XC (2009) Relationship between the impacted mandibular third molar and the mandibular canal on panoramic radiograph and cone beam computed tomography. Zhonghua Kou Qiang Yi Xue Za Zhi 44, 217-221. (in Chinese)

4. Ghaeminia H, Meijer GJ, Soehardi A, Borstlap WA, Mulder J, Bergé SJ (2009) Position of the 
impacted third molar in relation to the mandibular canal. Diagnostic accuracy of cone beam computed tomography compared with panoramic radiography. Int J Oral Maxillofac Surg 38, 964-971.

5. Hamada $\mathrm{Y}$, Kondoh T, Noguchi $\mathrm{K}$, Iino M, Isono $\mathrm{H}$, Ishii H, Mishima A, Kobayashi K, Seto K (2005) Application of limited cone beam computed tomography to clinical assessment of alveolar bone grafting: a preliminary report. Cleft Palate Craniofac J 42, 128-137.

6. Tsiklakis K, Syriopoulos K, Stamatakis HC (2004) Radiographic examination of the temporomandibular joint using cone beam computed tomography. Dentomaxillofac Radiol 33, 196-201.

7. Ogawa T, Enciso R, Memon A, Mah JK, Clark GT (2005) Evaluation of 3D airway imaging of obstructive sleep apnea with cone-beam computed tomography. Stud Health Technol Inform 111, 365-368.

8. Hassan B, Metska ME, Ozok AR, van der Stelt P, Wesselink PR (2009) Detection of vertical root fractures in endodontically treated teeth by a cone beam computed tomography scan. J Endod 35, 719-722.

9. Grimard BA, Hoidal MJ, Mills MP, Mellonig JT, Nummikoski PV, Mealey BL (2009) Comparison of clinical, periapical radiograph, and cone-beam volume tomography measurement techniques for assessing bone level changes following regenerative periodontal therapy. J Periodontol 80, 48-55.

10. Maki K, Inou N, Takanishi A, Miller AJ (2003) Computer-assisted simulations in orthodontic diagnosis and the application of a new cone beam X-ray computed tomography. Orthod Craniofac Res 6, Supp1 1, 95-101.

11. Hatcher DC, Dial C, Mayorga C (2003) Cone beam CT for pre-surgical assessment of implant sites. J Calif Dent Assoc 31, 825-833.

12. Kumar V, Gossett L, Blattner A, Iwasaki LR, Williams K, Nickel JC (2011) Comparison between cone-beam computed tomography and intraoral digital radiography for assessment of tooth root lesions. Am J Orthod Dentofacial Orthop 139, e533-541.

13. Fornell J, Johansson LA, Bolin A, Isaksson S, Sennerby L (2012) Flapless, CBCT-guided osteotome sinus floor elevation with simultaneous implant installation. I: radiographic examination and surgical technique. A prospective 1-year follow-up. Clin Oral Implants Res 23, 28-34.

14. Shiratori LN, Marotti J, Yamanouchi J, Chilvarquer
I, Contin I, Tortamano-Neto P (2012) Measurement of buccal bone volume of dental implants by means of cone-beam computed tomography. Clin Oral Implants Res 23, 797-804.

15. Wong RW, Chau AC, Hägg U (2011) 3D CBCT McNamara's cephalometric analysis in an adult southern Chinese population. Int J Oral Maxillofac Surg 40, 920-925.

16. Schulze RK, Berndt D, d'Hoedt B (2010) On conebeam computed tomography artifacts induced by titanium implants. Clin Oral Implants Res 21, 100-107.

17. Bauman R, Scarfe W, Clark S, Morelli J, Scheetz J, Farman A (2011) Ex vivo detection of mesiobuccal canals in maxillary molars using $\mathrm{CBCT}$ at four different isotropic voxel dimensions. Int Endod $\mathrm{J}$ 44, 752-758.

18. Maloul A, Fialkov J, Whyne C (2011) The impact of voxel size-based inaccuracies on the mechanical behavior of thin bone structures. Ann Biomed Eng 39, 1092-1100.

19. Matsubara K, Koshida K, Suzuki M, Hayashi N, Takata T, Tsujii H, Yamamoto T, Matsui O (2008) Contrast resolution in multidetector-row CT with 16 detector rows: phantom study. Radiol Phys Technol 1, 13-19.

20. Kalender WA, Deak P, Kellermeier M, van Straten M, Vollmar SV (2009) Application- and patient size-dependent optimization of x-ray spectra for CT. Med Phys 36, 993-1007.

21. White DR, Martin RJ, Darlison R (1977) Epoxy resin based tissue substitutes. Br J Radiol 50, 814-821.

22. Bechara BB, Moore WS, McMahan CA, Noujeim M (2012) Metal artifact reduction with cone beam CT: an in-vitro study. Dentomaxillofac Radiol 41, 248-253.

23. Bechara B, McMahan CA, Geha H, Noujeim M (2012) Evaluation of a cone beam CT artefact reduction algorithm. Dentomaxillofac Radiol 41, 422-428.

24. Draper NR, Smith H (1998) Applied regression analysis. 3rd ed, John Wiley \& Sons, New York.

25. Lofthag-Hansen S, Thilander-Klang A, Gröndahl K (2011) Evaluation of subjective image quality in relation to diagnostic task for cone beam computed tomography with different fields of view. Eur J Radiol 80, 483-488.

26. Tanimoto H, Arai Y (2009) The effect of voxel size on image reconstruction in cone-beam computed tomography. Oral radiology 25, 149-153. 